Clinical Research Paper

\title{
Oxaliplatin, 5-fluorouracil and leucovorin (FOLFOX) as second- line therapy for patients with advanced urothelial cancer
}

\author{
Sheng Zhang', Hongxi Xue' ${ }^{2}$, Qiang Chen ${ }^{3}$ \\ ${ }^{1}$ Medical Oncology, Fudan University Shanghai Cancer Center, Department of Oncology, Shanghai Medical College, Fudan \\ University, Shanghai, China \\ ${ }^{2}$ Rizhao City Hospital of Traditional Chinese Medicine, Rizhao, China \\ ${ }^{3}$ Department of Clinical Biochemistry, School of Public Health, Taishan Medical University, Tai'an, China \\ Correspondence to: Sheng Zhang, email: wozhangsheng@hotmail.com \\ Keywords: urothelial cancer, oxaliplatin, leucovorin, 5-fluorouracil, clinical trial \\ Received: February 08, 2016 \\ Accepted: June 30, 2016 \\ Published: July 07, 2016
}

\section{ABSTRACT}

There is currently no standard treatment for metastatic urothelial cancer after failure of cisplatin-based therapy. The present retrospective study investigated the efficacy and safety of oxaliplatin plus 5-fluorouracil (5-FU) and leucovorin (LV) (FOLFOX) in locally advanced or metastatic urothelial cancer patients following cisplatin-based treatment. Thirty-three patients who had received one or two cisplatin-based regimens were treated with oxaliplatin $\left(85 \mathrm{mg} / \mathrm{m}^{2}\right)$ as a $2-\mathrm{h}$ infusion on day 1, LV $\left(200 \mathrm{mg} / \mathrm{m}^{2}\right)$ as a 2-h infusion followed by bolus $5-\mathrm{FU}\left(400 \mathrm{mg} / \mathrm{m}^{2}\right)$ on day 1 , or a 44-h continuous $5-F U\left(1,200 \mathrm{mg} / \mathrm{m}^{2}\right)$ infusion. Patients were a mean of 67 years old with two involved organs. Metastases were mostly in the lung $(43 \%)$, lymph nodes $(51 \%)$ and liver (46\%). Based on an intention-to-treat analysis, nine patients achieved a partial response, with an overall response rate of $27 \%$. Eight (24\%) patients had stable disease. Mean progression-free survival was 3 months and mean overall survival was 6.1 months. Toxicity was mild to moderate with grade 3 or 4 neutropenia, thrombocytopenia and neuropathy occurring in $5(15 \%), 4(12 \%)$ and $2(6 \%)$ patients, respectively. This study demonstrated that oxaliplatin plus 5-FU/LV was a well-tolerated second-line regimen with moderate activity in patients pretreated with cisplatin-based therapeutics.

\section{INTRODUCTION}

Urothelial cancer is the sixth most common cancer and accounts for more than 13,000 deaths annually [1]. Cisplatin-based chemotherapy is the standard of care for patients with metastatic or advanced disease [2], and about $50 \%$ of patients achieve an objective response. Methotrexate, vinblastine, doxorubicin and cisplatin (MVAC) or the combination of gemcitabine and cisplatin have become first-line treatment standards, based on results from randomized phase III trials [3, 4]. For patients who complete one of these cisplatin-based regimens, there is no standard second-line chemotherapy regimen. Under these circumstances, many single agents, including docetaxel, paclitaxel and pemetrexed, demonstrated moderate responses (between 10 and 20\%) mainly in phase II studies, yet no drug has prolonged overall survival (OS) in randomized settings [5-8]. Vinflunine is a novel synthetic vinca alkaloid. In a phase III trial, cisplatin-refractory patients with metastatic or advanced urothelial cancer received vinflunine or best supportive care. Vinflunine did not confer an OS advantage across the trial population and consequently is not approved for patient use in the United States [9, 10]. However, OS advantage was demonstrated when only eligible patients were considered, and vinflunine was approved in Europe.

The lack of effective alternative treatment options for patients on cisplatin-based regimens emphasizes the need for novel therapeutics. In addition, impaired renal function in urothelial cancer patients compounds patient management challenges. Oxaliplatin has shown promising activity in metastatic urothelial patients in a few early trials [11]. When 37 adult cancer patients received oxaplatin in a phase I trial, no dose-limiting toxicities were observed in patients with creatine clearance $\geq 20 \mathrm{ml} / \mathrm{min}$. This suggests that oxaliplatin therapy is well tolerated by patients with 
mild to moderate renal dysfunction [12]. Oxaliplatin has also been used extensively with 5-fluorouracil (5-FU) and leucovorin (LV) in the treatment of colorectal cancer and others [13]. 5-FU was moderately active in the treatment of urothelial cancer patients in a pilot study [14]. On the basis of these encouraging data, we applied biweekly oxaliplatin therapy with LV and continuous 5-FU infusion in patients with urothelial cancer after failure of cisplatinbased therapy.

\section{RESULTS}

\section{Patient characteristics and results}

Thirty-three patients with locally advanced or metastatic urothelial cancer between January 2008 and March 2011 were selected for this study (Table 1). Mean patient age was 67 years (from 48 to 82 years) and two organs were involved on average (from one to five). Metastases were found mostly in the lung (43\%), lymph nodes $(51 \%)$ and liver (46\%). Patients had received 1.4 prior cisplatin-based chemotherapy regimens on average (from one to two). Fifty-seven percent of patients responded to previous cisplatin-based chemotherapy treatments, while $43 \%$ did not.

Of the 33 patients, none achieved $\mathrm{CR}$ and nine achieved PR during the study. The overall response rate was $27 \%$, eight (24\%) patients had SD and the CBR rate was $51 \%$ (Table 2). Mean PFS was three months $(95 \% \mathrm{CI}$, 2.5 to 3.5 , Figure 1), and mean OS was 6.1 months $(95 \%$ CI, 4.2 to 8.3 , Figure 2).

\section{Treatment regimen and toxicity}

161 chemotherapy cycles were administered at an average of 3.5 cycles per patient (from 1-10). Toxicity was generally mild to moderate (Table 3), and the most common type of toxicity was hematological, with neutropenia and thrombocytopenia. Grade 3 or higher neutropenia, thrombocytopenia and neuropathy occurred in five $(15 \%)$, four $(12 \%)$, and two $(6 \%)$ patients, respectively. Manageable gastrointestinal toxicity was reported, with grade 3 nausea and vomiting in only one patient. Nine $(27 \%)$ patients needed at least one dose reduction, most commonly due to neutropenia $(9 \%)$ or neuropathy $(6 \%)$. No treatment-related deaths occurred.

\section{DISCUSSION}

Given that management of metastatic urothelial cancer remains a formidable challenge, a clear and unmet clinical need for novel therapies remains for patients resistant to cisplatin-based treatments [15-17]. In the present report, an oxaliplatin and 5-FU/LV (FOLFOX) treatment regimen was used in the management of urothelial cancer patients after failure of cisplatin-based treatment. With this regimen, patients with locally advanced or metastatic urothelial cancer, who had been pretreated with cisplatin-containing agents, achieved a $27 \%$ ORR and $51 \%$ CBR. Most previous studies evaluating multiple regimens achieved ORRs between 10 and $20 \%$ [16].

While our results were encouraging, our study might have been subject to certain biases common to retrospective studies, such as selection bias. Thus our results should be interpreted with caution. Our results appear consistent with those of several other small phase 2 studies $[17,18]$. A phase 2 study of the folate antagonist pemetrexed showed a similar ORR of $27 \%$ [7]. Similarly, when 48 metastatic urothelial cancer patients were treated with nanoparticle albumin-bound paclitaxel at three-week intervals, a 28\% ORR was achieved [19]. The single agent pazopanib was the first targeted agent to show significant clinical activity in previously treated urothelial cancer patients, with an ORR of 17\% [20].

Rosenberg, et al. recently reported the results of an multicenter phase 2 study of atezolizumab in patients with locally advanced or metastatic urothelial carcinoma [21]. Compared with the historical ORR of $10 \%$, atezolizumab therapy resulted in a significantly higher rate of $15 \%$. Importantly, higher ORRs were achieved in individuals expressing higher programmed death-ligand 1 (PD-L1) levels. This finding must be confirmed in ongoing phase III trials.

The mean OS of 6.1 months was shorter than that of pemetrexed and docetaxel $[7,8]$. It is possible that our study selected patients with relatively poor prognoses, as compared with other similar studies. Eighty-two percent of our patients had visceral metastases, and less than half had a PS of 0. Both poor PS and visceral (especially hepatic) metastases were independent negative prognostic factors in a phase 3 vinflunine study [9]. Our results were consistent with these findings.

Patients with advanced urothelial cancer after cisplatin-based treatment are generally older and more fragile, with possible poor PS and impaired renal function. For this reason, the majority of clinical trials in metastatic urothelial cancer are small phase 2 trials [22]. Our results provided an alternative second or third-line metastatic urothelial cancer therapy option, following failure of cisplatin-based treatment, and should be tested in prospective trials. Importantly, the oxaliplatin-based regimen described here does not require dose adjustments for patients with mild to moderate renal dysfunction, favoring this regimen over other choices under these conditions.

The safety profile of the described treatment regimen was acceptable. Grades 3 and 4 neutropenia, thrombocytopenia and neurotoxicity were experienced by 15,12 and $6 \%$ of patients, respectively, and nine (27\%) patients required dose reductions. Additional adverse responses included anemia, nausea and vomiting, diarrhea and fever, and no treatment-related deaths occurred. 
Table 1: Patient Characteristics $(\mathrm{N}=33)$

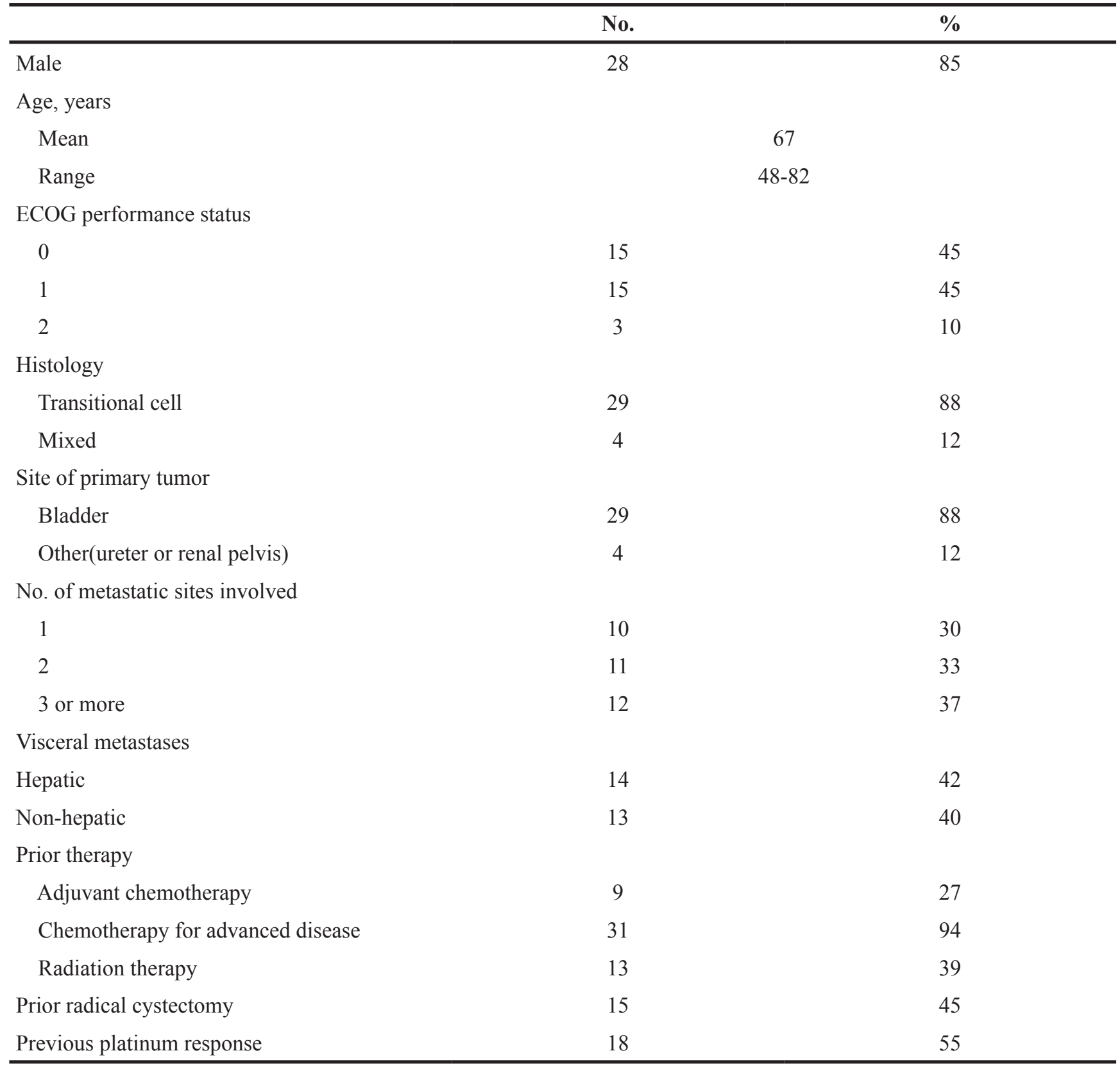

Abbreviation:ECOG, Eastern Cooperative Oncology Group.

Table 2: Response to FOLOFX regimen $(\mathrm{N}=33)$

\begin{tabular}{lcc}
\hline & No. of patients & \% \\
\hline Response & & \\
Complete response & 0 & 0 \\
Partial response & 9 & 27 \\
Stable disease & 8 & 24 \\
Overall response & 9 & 27 \\
Progressive disease & 16 & 48 \\
Clinical benefit rate & 17 & 52 \\
\hline
\end{tabular}




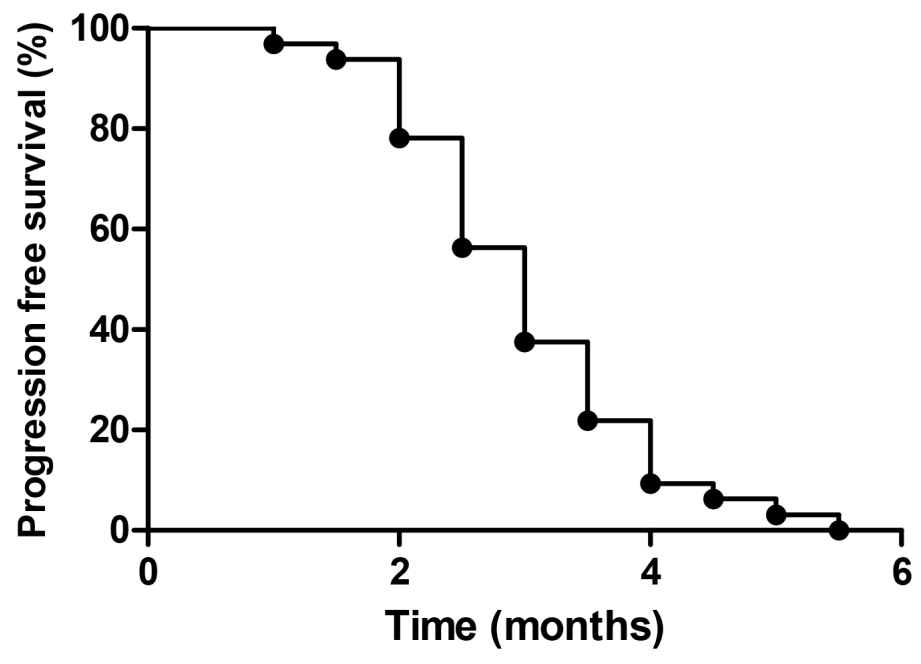

Figure 1: Profession-free survival among all patients.

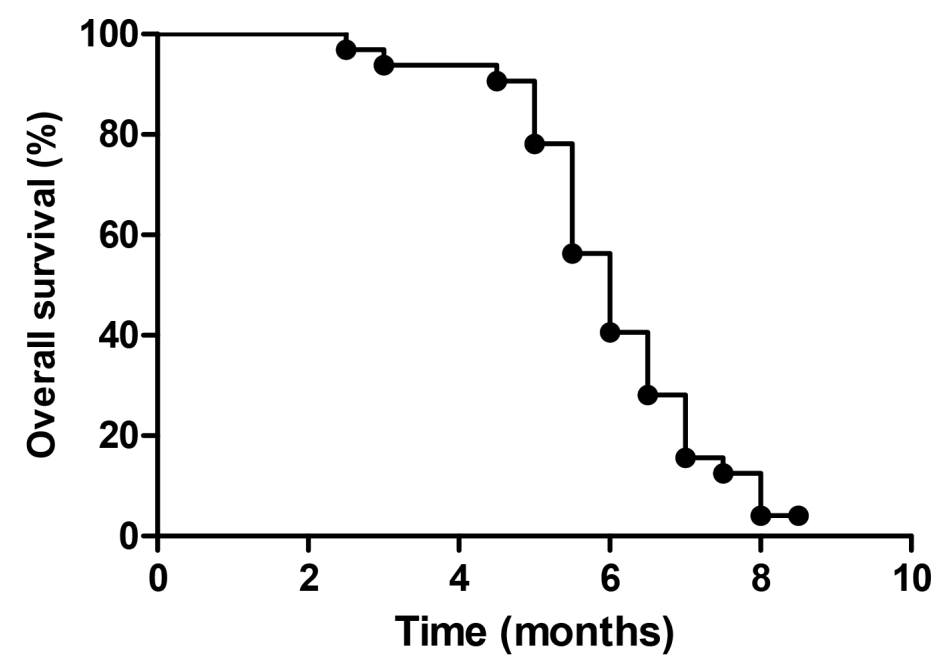

Figure 2: Overall survival among all patients.

Table 3: Toxicities of patients

\begin{tabular}{lcccc}
\hline Toxicity & \multicolumn{5}{c}{ Grade, n (\%) } \\
\cline { 2 - 5 } & $\mathbf{1}$ & $\mathbf{2}$ & $\mathbf{3}$ & $\mathbf{4}$ \\
\hline Anemia & $13(39)$ & $3(9)$ & 0 & 0 \\
Neutropenia & $11(33)$ & $4(12)$ & $3(9)$ & $2(6)$ \\
Thrombocytopenia & $6(18)$ & $3(9)$ & $2(6)$ & $2(6)$ \\
Nausea/vomiting & $5(15)$ & $4(12)$ & $1(3)$ & 0 \\
Diarrhea & $2(6)$ & $1(3)$ & 0 & 0 \\
Fever & $3(9)$ & $1(3)$ & 0 & 0 \\
Neuropathy & $6(18)$ & $1(3)$ & $2(6)$ & 0 \\
\hline
\end{tabular}


In conclusion, in patients for whom cisplatinbased treatment has failed, oxaliplatin, LV and 5-FU in combination are well tolerated and demonstrate clinically meaningful activity. Treatment efficacy should be further confirmed in prospective, and ideally randomized, clinical trials.

\section{METHODS AND MATERIALS}

\section{Patients}

This study was a single-institution retrospective analysis within the Department of Internal Medicine of Shanghai Cancer Hospital, China. The study was approved by the institutional review board, and informed patient consent was waved because of the retrospective nature of the study.

Eligibility criteria included: (1) age 18 years or older, Eastern Cooperative Oncology Group (ECOG) performance status (PS) 0-2 and a life expectancy of more than 12 weeks; (2) histologically-diagnosed, measurable locally advanced or metastatic transitional carcinoma of the urinary bladder urothelium or upper urinary tract; (3) documented disease progression after first or secondline cisplatin-based treatment; (4) no prior treatment with 5-FU infusion and/or oxaliplatin therapy; (5) and adequate liver, renal (calculated creatinine clearance $\geq 30 \mathrm{ml} / \mathrm{min}$ by the Cockcroft-Gault formula), medullary and cardiac functions. Patients previously treated with radiotherapy were eligible for the study, provided that measurable disease existed outside the radiation field. Patients with brain metastases were eligible provided that they had received cranial irradiation with clinical and radiological improvement of their central nervous system disease. Patients were excluded from the study if they had secondary malignancy (except for carcinoma of the skin) and preexisting motor or sensory neurotoxicity grade $\geq 2$, according to the Common Terminology Criteria for Adverse Events 3.0 (CTCAE 3.0) scale (intolerable paresthesias and/or marked motor loss). Fertile patients without the use of adequate contraceptive measures and pregnant or breast-feeding women were ineligible for the study. Patients with active infection or other serious underlying medical conditions that would impair their ability to receive the treatment or those without appropriate medical files were also excluded from the study.

\section{Study design}

Oxaliplatin was administered at $85 \mathrm{mg} / \mathrm{m}^{2}$ in $5 \%$ glucose as a 2-h infusion on day 1, LV $200 \mathrm{mg} / \mathrm{m}^{2}$ as a 2-h infusion followed by bolus $5-\mathrm{FU} 400 \mathrm{mg} / \mathrm{m}^{2}$ on day 1, and a 44-h infusion of 5-FU 1,200 mg/m². Treatment was repeated every two weeks. Patients were evaluated for response usually every eight weeks. Patients were premedicated with antiemetics, including 5-hydroxytryptamine-3 receptor antagonists and corticosteroids. Hematopoietic growth factors and transfusion were allowed. Treatment was continued until disease progression, unacceptable toxicity, death or withdrawal of informed consent.

Oxaliplatin and 5-FU doses were reduced by $25 \%$ in patients who experienced dose-limiting toxicity (DLT), defined as grade 4 neutropenia lasting for more than seven days, febrile neutropenia, grade 4 thrombocytopenia, grade 3 thrombocytopenia associated with bleeding or grades 3-4 non-hematological toxicities (except alopecia and neuropathy). In the presence of grade 3/4 neurotoxicity, treatment was delayed until recovery to toxicity grade $\leq 1$ (no longer than 14 days). These patients continued to receive reduced doses in subsequent cycles for the remainder of the study. Two dose reductions were allowed. These patients were discontinued from the study if there was evidence of disease progression, presence of unacceptable toxicity, interruption of treatment for more than two weeks, withdrawal of informed consent or if a third dose reduction was required.

\section{Study assessment}

Pretreatment evaluation included a complete medical history and physical examination, hematological and biochemical profiles, electrocardiography (ECG), and computed scan of the chest, abdomen and pelvis. During the treatment period, complete blood counts were performed weekly or every two days in cases of grade 3/4 neutropenia or thrombocytopenia until hematological recovery occurred. Efficacy was evaluated in patients who received at least two chemotherapy cycles. Patients were evaluated for response according to the National Cancer Institute's (US) response evaluation criteria in solid tumors (RECIST). Complete response (CR) was defined as the disappearance of all known lesions and normalization of tumor marker levels for at least four weeks. Partial response (PR) was defined as a reduction in the sum of all measurable lesions by at least $30 \%$ for at least four weeks. Progressive disease (PD) was defined as an increase in the sum of all measurable lesions by $>20 \%$ or the appearance of a new lesion, and stable disease (SD) was defined as neither CR, PR nor PD. Overall response rate (ORR) was defined as the sum of $C R$ and $\mathrm{PR}$ rates. Clinical benefit rate (CBR) was defined as the sum of CR, PR and long SD rates. In patients with tumor response or stable disease, the treatment was continued for up to 8-12 cycles; thereafter, maintenance therapy was based on the clinician's decision. After completion of the treatment period, the patients were followed up every 1.5 months. All adverse events were graded according to the National Cancer Institute's Common Toxicity Criteria, version 3.0. 


\section{Statistical analysis}

All statistical analyses were carried out on an intention-to treat basis with SPSS 17.0 software (Chicago, Illinois, USA). Progression-free survival (PFS) was calculated for all assessable patients as the time from inclusion to disease progression or death from any cause. Overall survival (OS) was calculated for all patients from the date of inclusion until death. PFS and OS were computed using the Kaplan-Meier method

\section{ACKNOWLEDGMENTS}

We thank all patients and their families who participated in this study.

\section{CONFLICT OF INTEREST}

The authors declare no conflicts of interest.

\section{REFERENCES}

1. Siegel R, Naishadham D, Jemal A. Cancer statistics, 2013. CA Cancer J Clin. 2013; 63: 11-30. doi: 10.3322/ caac. 21166.

2. Galsky MD, Chen GJ, Oh WK, Bellmunt J, Roth BJ, Petrioli R, Dogliotti L, Dreicer R, Sonpavde G. Comparative effectiveness of cisplatin-based and carboplatin-based chemotherapy for treatment of advanced urothelial carcinoma. Ann Oncol. 2012; 23: 406-10. doi: 10.1093/ annonc/mdr 156 .

3. von der Maase H, Hansen SW, Roberts JT, Dogliotti L, Oliver T, Moore MJ, Bodrogi I, Albers P, Knuth A, Lippert CM, Kerbrat P, Sanchez Rovira P, Wersall P, et al. Gemcitabine and cisplatin versus methotrexate, vinblastine, doxorubicin, and cisplatin in advanced or metastatic bladder cancer: results of a large, randomized, multinational, multicenter, phase III study. J Clin Oncol. 2000; 18: 3068-77.

4. von der Maase H, Sengelov L, Roberts JT, Ricci S, Dogliotti L, Oliver T, Moore MJ, Zimmermann A, Arning M. Longterm survival results of a randomized trial comparing gemcitabine plus cisplatin, with methotrexate, vinblastine, doxorubicin, plus cisplatin in patients with bladder cancer. J Clin Oncol. 2005; 23: 4602-8. doi: 10.1200/ jco.2005.07.757.

5. McCaffrey JA, Hilton S, Mazumdar M, Sadan S, Kelly WK, Scher HI, Bajorin DF. Phase II trial of docetaxel in patients with advanced or metastatic transitional-cell carcinoma. J Clin Oncol. 1997; 15: 1853-7.

6. Vaughn DJ, Broome CM, Hussain M, Gutheil JC, Markowitz AB. Phase II trial of weekly paclitaxel in patients with previously treated advanced urothelial cancer. J Clin Oncol. 2002; 20: 937-40.
7. Sweeney CJ, Roth BJ, Kabbinavar FF, Vaughn DJ, Arning M, Curiel RE, Obasaju CK, Wang Y, Nicol SJ, Kaufman DS. Phase II study of pemetrexed for second-line treatment of transitional cell cancer of the urothelium. J Clin Oncol. 2006; 24: 3451-7. doi: 10.1200/jco.2005.03.6699.

8. Choueiri TK, Ross RW, Jacobus S, Vaishampayan U, Yu EY, Quinn DI, Hahn NM, Hutson TE, Sonpavde G, Morrissey SC, Buckle GC, Kim WY, Petrylak DP, et al. Doubleblind, randomized trial of docetaxel plus vandetanib versus docetaxel plus placebo in platinum-pretreated metastatic urothelial cancer. J Clin Oncol. 2012; 30: 507-12. doi: 10.1200/jco.2011.37.7002.

9. Bellmunt J, Theodore C, Demkov T, Komyakov B, Sengelov L, Daugaard G, Caty A, Carles J, Jagiello-Gruszfeld A, Karyakin O, Delgado FM, Hurteloup P, Winquist E, et al. Phase III trial of vinflunine plus best supportive care compared with best supportive care alone after a platinumcontaining regimen in patients with advanced transitional cell carcinoma of the urothelial tract. J Clin Oncol. 2009; 27: 4454-61. doi: 10.1200/jco.2008.20.5534.

10. Bellmunt J, Fougeray R, Rosenberg JE, von der Maase H, Schutz FA, Salhi Y, Culine S, Choueiri TK. Long-term survival results of a randomized phase III trial of vinflunine plus best supportive care versus best supportive care alone in advanced urothelial carcinoma patients after failure of platinum-based chemotherapy. Ann Oncol. 2013; 24: 146672. doi: 10.1093/annonc/mdt007.

11. Carles J, Esteban E, Climent M, Font A, Gonzalez-Larriba JL, Berrocal A, Garcia-Ribas I, Marfa X, Fabregat X, Albanell J, Bellmunt J. Gemcitabine and oxaliplatin combination: a multicenter phase II trial in unfit patients with locally advanced or metastatic urothelial cancer. Ann Oncol. 2007; 18: 1359-62. doi: 10.1093/annonc/mdm160.

12. Takimoto $\mathrm{CH}$, Remick SC, Sharma S, Mani S, Ramanathan RK, Doroshow J, Hamilton A, Mulkerin D, Graham M, Lockwood GF, Ivy P, Egorin M, Schuler B, et al. Doseescalating and pharmacological study of oxaliplatin in adult cancer patients with impaired renal function: a National Cancer Institute Organ Dysfunction Working Group Study. J Clin Oncol. 2003; 21: 2664-72. doi: 10.1200/ jco.2003.11.015.

13. Sun S, Wang LP, Zhang J, Yang XY, Zhang QL, Jia Z, Hu $X C$, Wang BY. Phase II study of oxaliplatin plus leucovorin and 5-fluorouracil in heavily pretreated metastatic breast cancer patients. Med Oncol. 2012; 29: 418-24. doi: 10.1007/ s12032-011-9839-6.

14. Di Lorenzo G, Autorino R, Giordano A, Giuliano M, D'Armiento M, Bianco AR, De Placido S. FOLFOX-4 in pre-treated patients with advanced transitional cell carcinoma of the bladder. Jpn J Clin Oncol. 2004; 34: 74750. doi: 10.1093/jjco/hyh132.

15. Dreicer R. The future of drug development in urothelial cancer. J Clin Oncol. 2012; 30: 473-5. doi: 10.1200/ jco.2011.39.5566. 
16. Sonpavde G, Rosenberg JE, Hahn NM, Galsky MD, Bangs R, Sternberg CN, Vogelzang NJ. Suggestions for regulatory agency approval of second-line systemic therapy for metastatic transitional cell carcinoma. J Clin Oncol. 2010; 28: e205-7; author reply e8. doi: 10.1200/jco.2009.27.1114.

17. Dreicer R. Second-line chemotherapy for advanced urothelial cancer: because we should or because we can? J Clin Oncol. 2009; 27: 4444-5. doi: 10.1200/ jco.2009.23.8071.

18. Rozzi A, Santini D, Salerno M, Bordin F, Mancuso A, Minniti G, Nardoni C, Corona M, Falbo PT, Recine F, Lanzetta G. Pegylated liposomal doxorubicin as thirdline chemotherapy in patients with metastatic transitional cell carcinoma of urothelial tract: results of a phase II study. Med Oncol. 2013; 30: 407. doi: 10.1007/ s12032-012-0407-5.

19. Ko YJ, Canil CM, Mukherjee SD, Winquist E, Elser C, Eisen A, Reaume MN, Zhang L, Sridhar SS. Nanoparticle albumin-bound paclitaxel for second-line treatment of metastatic urothelial carcinoma: a single group, multicentre, phase 2 study. Lancet Oncol. 2013; 14: 769-76. doi: 10.1016/s1470-2045(13)70162-1.
20. Necchi A, Mariani L, Zaffaroni N, Schwartz LH, Giannatempo P, Crippa F, Morosi C, Lanocita R, Sava T, Ortega C, Messina C, Sacco C, Pennati M, et al. Pazopanib in advanced and platinum-resistant urothelial cancer: an open-label, single group, phase 2 trial. Lancet Oncol. 2012; 13: 810-6. doi: 10.1016/s1470-2045(12)70294-2.

21. Rosenberg JE, Hoffman-Censits J, Powles T, van der Heijden MS, Balar AV, Necchi A, Dawson N, O'Donnell PH, Balmanoukian A, Loriot Y, Srinivas S, Retz MM, Grivas $\mathrm{P}$, et al. Atezolizumab in patients with locally advanced and metastatic urothelial carcinoma who have progressed following treatment with platinum-based chemotherapy: a single-arm, multicentre, phase 2 trial. Lancet. 2016. doi: 10.1016/s0140-6736(16)00561-4.

22. Galsky MD, Hendricks R, Svatek R, Bangs R, HoffmanCensits J, Clement J, Dreicer R, Guancial E, Hahn N, Lerner SP, O'Donnell PH, Quale DZ, Siefker-Radtke A, et al. Critical analysis of contemporary clinical research in muscle-invasive and metastatic urothelial cancer: a report from the Bladder Cancer Advocacy Network Clinical Trials Working Group. Cancer. 2013; 119: 1994-8. doi: 10.1002/ cncr.27973. 